\title{
The phase structure of a chirally invariant lattice Higgs-Yukawa model
}

\section{Philipp Gerhold*}

Humboldt-Universität Berlin

E-mail: gerhold@physik.hu-berlin.de

\section{Karl Jansen}

DESY, Zeuthen

E-mail: Karl.Jansen@desy.de

\begin{abstract}
We consider a chirally invariant lattice Higgs-Yukawa model based on the Neuberger overlap operator $\mathscr{D}^{(o v)}$. As a first step towards the eventual determination of Higgs mass bounds we present the phase structure of the model analytically in the large $N_{f}$-limit in the physically interesting region of the Yukawa coupling constant. We confront the analytically obtained phase diagram with corresponding HMC-simulations and find an excellent agreement at large values of $N_{f}$. In the opposite case the large $N_{f}$ computation still gives a good qualitative description of the phase diagram. We also present first and very preliminary results on the Higgs upper bound at one selected cut-off $\Lambda$.
\end{abstract}

The XXV International Symposium on Lattice Field Theory

July 30 - August 42007

Regensburg, Germany

${ }^{*}$ Speaker. 


\section{Introduction}

The main target of lattice studies of the Higgs-Yukawa sector of the electroweak standard model is the non-perturbative determination of the $\Lambda$-dependence of the upper and lower bounds of the Higgs boson mass $[1,2]$ as well as its decay properties, where $\Lambda$ denotes the cut-off of the theory. There are two main developments which warrant to reconsider these questions: first, with the advent of the LHC, we are to expect that properties of the standard model Higgs boson, such as the mass and the decay width, will be revealed experimentally. Second, there is, in contrast to the situation of earlier investigations of lattice Higgs-Yukawa models [3, 4, 5, 6], a consistent formulation of an Higgs-Yukawa model with an exact lattice chiral symmetry [7] based on the Ginsparg-Wilson relation [8], which allows to establish a lattice version of chiral symmetry while lifting the unwanted fermion doublers at the same time.

Before addressing the questions of the Higgs mass bounds and decay properties, we started with an investigation of the phase structure of the model in order to obtain first information about the region of the (bare) couplings in parameter space where eventual simulations of phenomenological interest should be performed.

In the present paper we basically summarize some of the most important results of our work on the model's phase structure, which we have studied analytically in the large $N_{f}$-limit for small as well as for large values of the Yukawa coupling constant [9], and numerically by means of HMCsimulations [10]. Finally, we give a brief outlook towards some first and very preliminary results on the upper Higgs boson mass obtained at one selected cut-off $\Lambda$.

\section{The model and its numerical treatment}

The model, we consider here, is a four-dimensional, chirally invariant $S U(2)_{L} \times S U(2)_{R}$ HiggsYukawa model discretized on a finite lattice with $L$ lattice sites per dimension. The model contains one four-component, real Higgs field $\Phi$ and $N_{f}$ fermion doublets represented by eight-component spinors $\psi^{(i)}, \bar{\psi}^{(i)}, i=1, \ldots, N_{f}$ with the total action being decomposed into the Higgs action $S_{\Phi}$, and the fermion action $S_{F}$. It should be stressed here that no gauge fields are included within this model.

The fermion action $S_{F}$ is based on the Neuberger overlap operator $\mathscr{D}^{(o v)}$ [11] and can be written as

$$
S_{F}=\sum_{i=1}^{N_{f}} \bar{\psi}^{(i)}[\underbrace{\mathscr{D}^{(o v)}+y_{N} B \cdot\left(\mathbb{1}-\frac{1}{2 \rho} \mathscr{D}^{(o v)}\right)}_{\mathscr{M}}] \psi^{(i)}, \quad B_{n, m}=\mathbb{1}_{n, m}\left[\frac{\left(1-\gamma_{5}\right)}{2} \phi_{n}+\frac{\left(1+\gamma_{5}\right)}{2} \phi_{n}^{\dagger}\right] .
$$

It describes the propagation of the fermion fields as well as their coupling to the Higgs field $\Phi$ through the Yukawa coupling matrix $B_{n, m}$ and the Yukawa coupling constant $y_{N}$. Here the Higgs field $\Phi_{n}$ was rewritten as a quaternionic, $2 \times 2$ matrix $\phi_{n}=\Phi_{n}^{0} \mathbb{1}-i \Phi_{n}^{j} \tau_{j}$, with $\vec{\tau}$ denoting the vector of Pauli matrices, acting on the $S U(2)$ index of the fermionic doublets.

Note that in absence of gauge fields the Neuberger Dirac operator can be trivially constructed in momentum space, since for that case its eigenvalues $v^{\varepsilon}(p), \varepsilon= \pm 1$ for the allowed four- 
component momenta $p \in \mathscr{P}$ are explicitly known. This will be exploited in the numerical construction of the overlap operator.

The model then obeys an exact, but lattice modified, chiral symmetry according to

$$
\delta \psi^{(i)}=i \varepsilon \gamma_{5}\left(1-\frac{1}{\rho} \mathscr{D}^{(o v)}\right) \psi^{(i)}, \quad \delta \phi=2 i \varepsilon \phi, \quad \delta \bar{\psi}^{(i)}=i \varepsilon \bar{\psi}^{(i)} \gamma_{5}, \quad \delta \phi^{\dagger}=-2 i \varepsilon \phi^{\dagger}
$$

recovering the chiral symmetry in the continuum limit [7].

The lattice Higgs action $S_{\Phi}$ is given by the usual lattice notation

$$
S_{\Phi}=-\kappa_{N} \sum_{n, \mu} \Phi_{n}^{\dagger}\left[\Phi_{n+\hat{\mu}}+\Phi_{n-\hat{\mu}}\right]+\sum_{n} \Phi_{n}^{\dagger} \Phi_{n}+\lambda_{N} \sum_{n}\left(\Phi_{n}^{\dagger} \Phi_{n}-N_{f}\right)^{2}
$$

with the only particularity that the fermion generation number $N_{f}$ appears in the quartic coupling term which is a convenient convention for the large $N_{f}$ analysis. However, this version of the lattice Higgs action is equivalent to the usual continuum notation [10].

For the numerical treatment of the model we have implemented an Hybrid-Monte-Carlo (HMC) algorithm for even values of $N_{f}$ with $N_{f} / 2$ complex pseudo-fermionic fields $\omega_{j}$ according to the HMC-Hamiltonian

$$
H\left(\Phi, \xi, \omega_{j}\right)=S_{\Phi}[\Phi]+\frac{1}{2} \xi^{\dagger} \xi+\sum_{j=1}^{N_{f} / 2} \frac{1}{2} \omega_{j}^{\dagger}\left[\mathscr{M} \mathscr{M}^{\dagger}\right]^{-1} \omega_{j}
$$

where $\xi$ denotes the real momenta, conjugate to the Higgs field $\Phi$. Since we focus here on checking the validity of our analytical investigation of the phase structure, which was determined in the large $N_{f}$-limit, the restriction to even $N_{f}$ does no harm. For the further details of this HMC algorithm we refer the interested reader to Ref. [10].

The observables we will be using for exploring the phase structure are the magnetization $m$ and the staggered magnetization $s$,

$$
m=\left[\sum_{i=0}^{3}\left|\frac{1}{L^{4}} \sum_{n} \Phi_{n}^{i}\right|^{2}\right]^{\frac{1}{2}}, \quad s=\left[\sum_{i=0}^{3}\left|\frac{1}{L^{4}} \sum_{n}(-1)^{\sum_{\mu} n_{\mu}} \cdot \Phi_{n}^{i}\right|^{2}\right]^{\frac{1}{2}}
$$

and the corresponding susceptibilities $\chi_{m}=L^{4} \cdot\left[\left\langle m^{2}\right\rangle-\langle m\rangle^{2}\right]$ and $\chi_{s}=L^{4} \cdot\left[\left\langle s^{2}\right\rangle-\langle s\rangle^{2}\right]$, where $\langle\ldots\rangle$ denotes the average over the $\Phi$-field configurations generated in the Monte-Carlo process.

To locate the phase transition points, we decided to fit the data for the susceptibilities $\chi_{m}, \chi_{s}$ as a function of $\kappa_{N}$ according to the - partly phenomenologically motivated - ansatz

$$
\chi_{m, s}=A_{1}^{m, s} \cdot\left(\frac{1}{L^{-2 / v}+A_{2,3}^{m, s}\left(\kappa_{N}-\kappa_{\mathrm{crit}}^{m, s}\right)^{2}}\right)^{\gamma / 2}
$$

where $A_{1}^{m, s}, A_{2,3}^{m, s}$, and $\kappa_{\text {crit }}^{m, s}$ are the fitting parameters for the magnetic susceptibility and staggered susceptibility, respectively, and $v, \gamma$ denote the critical exponents of the $\Phi^{4}$-theory. Here $A_{2,3}^{m}$ $\left(A_{2,3}^{s}\right)$ is actually meant to refer to two parameters, namely $A_{2}^{m}\left(A_{2}^{s}\right)$ for $\kappa_{N}<\kappa_{\text {crit }}^{m}\left(\kappa_{N}<\kappa_{\text {crit }}^{s}\right)$ and $A_{3}^{m}\left(A_{3}^{s}\right)$ in the other case, such that the resulting curve is not necessarily symmetric. The phase transition point is then given at the value of $\kappa_{N}=\kappa_{\text {crit }}^{m}\left(\kappa_{N}=\kappa_{\text {crit }}^{s}\right)$ where the magnetic (staggered) susceptibility develops its maximum. 


\section{Large $N_{f}$-limit for small Yukawa coupling parameters}

The phase structure of the considered Higgs-Yukawa model can be accessed in the large $N_{f^{-}}$ limit by scaling the coupling constants and the Higgs field itself according to

$$
y_{N}=\frac{\tilde{y}_{N}}{\sqrt{N_{f}}}, \quad \lambda_{N}=\frac{\tilde{\lambda}_{N}}{N_{f}}, \quad \kappa_{N}=\tilde{\kappa}_{N}, \quad \Phi_{n}=\sqrt{N_{f}} \cdot \tilde{\Phi}_{n},
$$

where the quantities $\tilde{y}_{N}, \tilde{\lambda}_{N}, \tilde{\kappa}_{N}$, and $\tilde{\Phi}_{n}$ are kept constant in the limit $N_{f} \rightarrow \infty$ allowing to factorize the fermion generation number $N_{f}$ out of the effective action $S_{\text {eff } f}[\Phi]=S_{\Phi}-N_{f} \log \operatorname{det}(\mathscr{M})$.

One is thus left with the problem of finding the absolute minima of $S_{\text {eff }}[\Phi]$ in terms of the latter quantities. For sufficiently small values of the Yukawa and quartic coupling constants the kinetic term of the Higgs action becomes dominant allowing to restrict the search for the absolute minima of $S_{\text {eff }}[\Phi]$ to the ansatz

$$
\Phi_{n}=\hat{\Phi} \cdot \sqrt{N_{f}} \cdot\left(\tilde{m}+\tilde{s} \cdot(-1)^{\sum_{\mu}^{\mu} n_{\mu}}\right), \quad \hat{\Phi} \in \mathbb{R}^{4}, \quad|\hat{\Phi}|=1
$$

taking only a magnetization $\tilde{m}$ and a staggered magnetization $\tilde{s}$ into account. After some work, which was presented in detail in [9], one finally finds for the effective action

$$
\begin{aligned}
S_{e f f}[\Phi] & =-N_{f} \cdot \sum_{p \in \mathscr{P}} \log \left[\left(\left|v^{+}(p)\right| \cdot\left|v^{+}(\wp)\right|+\frac{\tilde{y}_{N}^{2}}{4 \rho^{2}}\left(\tilde{m}^{2}-\tilde{s}^{2}\right) \cdot\left|v^{+}(p)-2 \rho\right| \cdot\left|v^{+}(\wp)-2 \rho\right|\right)^{2}\right. \\
& \left.+\tilde{m}^{2} \frac{\tilde{y}_{N}^{2}}{4 \rho^{2}}\left(\left|v^{+}(p)-2 \rho\right| \cdot\left|v^{+}(\wp)\right|-\left|v^{+}(\wp)-2 \rho\right| \cdot\left|v^{+}(p)\right|\right)^{2}\right]^{2}+S_{\Phi}[\Phi]
\end{aligned}
$$

while the Higgs action in this setting reads

$$
S_{\Phi}=N_{f} \cdot L^{4} \cdot\left\{-8 \tilde{\kappa}_{N}\left(\tilde{m}^{2}-\tilde{s}^{2}\right)+\tilde{m}^{2}+\tilde{s}^{2}+\tilde{\lambda}_{N}\left(\tilde{m}^{4}+\tilde{s}^{4}+6 \tilde{m}^{2} \tilde{s}^{2}-2\left(\tilde{m}^{2}+\tilde{s}^{2}\right)\right)\right\} \text {. }
$$

The resulting phase structure in the large $N_{f}$-limit can then be determined by minimizing the effective action with respect to $\tilde{m}$ and $\tilde{s}$. It is presented in Fig. 1a for the selected value of the quartic coupling constant $\tilde{\lambda}_{N}=0.1$ and $L=\infty$. Here we distinguish between the following four phases:

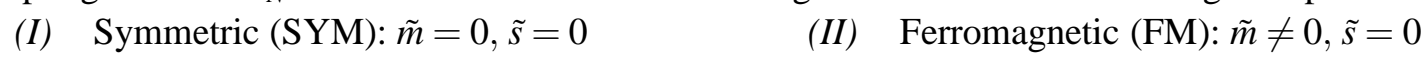

(III) Anti-ferromagnetic (AFM): $\tilde{m}=0, \tilde{s} \neq 0$

(IV) Ferrimagnetic (FI): $\tilde{m} \neq 0, \tilde{s} \neq 0$

In Fig. 1b we compare this analytically obtained $N_{f}=\infty, L=\infty$ phase structure with the results of corresponding HMC-simulations performed on $8^{4}$ - and $6^{4}$-lattices at $N_{f}=10$. As expected we observe a good qualitative agreement between the numerical and analytical results. On a quantitative level, however, the encountered deviations in Fig. $1 \mathrm{~b}$ need to be further addressed. These deviations can be ascribed to finite volume effects as well as finite $N_{f}$ corrections.

The finite size effects are illustrated in Fig. 2a, showing some phase transition points from the FM to the SYM phase as obtained from our numerical simulations on a $4^{4}$-lattice (open squares), and on an $8^{4}$-lattice (open circles) for the (very large) value of fermion generations $N_{f}=50$, chosen to isolate the finite size effects from the $1 / N_{f}$ corrections. One clearly observes that the phase transition line is shifted towards smaller values of the hopping parameter when the lattice size is increased. The numerical results are compared to the $N_{f}=\infty$ phase transition lines obtained for 


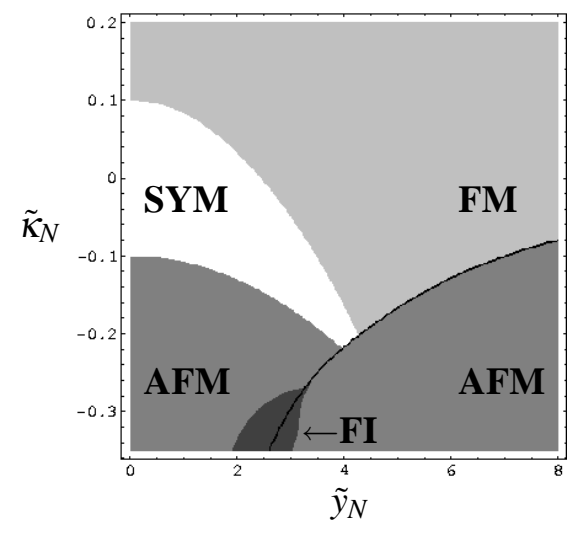

(a)

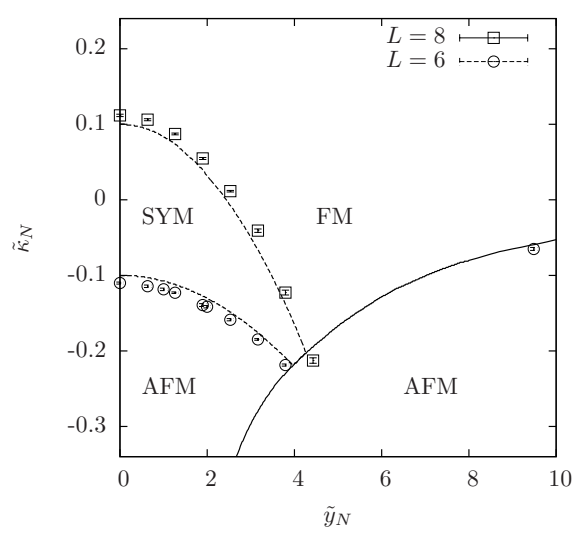

(b)

Figure 1: Phase diagrams with respect to the Yukawa coupling constant $\tilde{y}_{N}$ and the hopping parameter $\tilde{\kappa}_{N}$ for the constant quartic coupling $\tilde{\lambda}_{N}=0.1$. The black solid line indicates a first order phase transition, while the remaining transitions are of second order [10]. (a) Analytically obtained phase diagrams for $L=\infty$ and $N_{f}=\infty$. (b) Comparison with numerically obtained phase transition points for $N_{f}=10$ and $L^{4}=8^{4}$ (open squares) and $L^{4}=6^{4}$ (open circles).

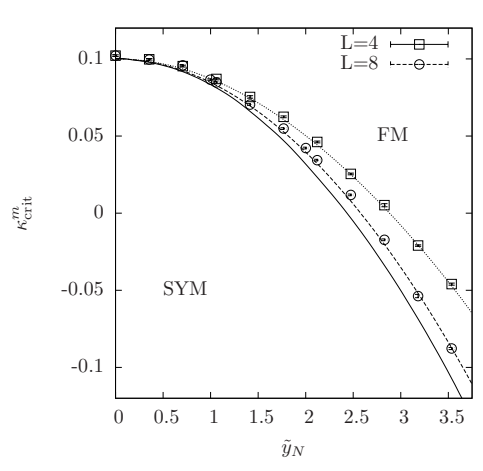

(a)

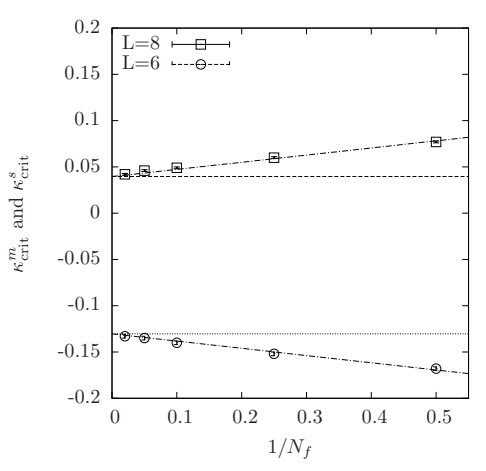

(b)

Figure 2: (a) Some selected phase transition points between the ferromagnetic and the symmetric phase, as obtained at $N_{f}=50$ on a $4^{4}$-lattice (open squares) and on an $8^{4}$-lattice (open circles), are compared to the $L=4$ (dotted), $L=8$ (dashed), and $L=\infty$ (solid) phase transition lines determined analytically in the large $N_{f}$-limit. (b) $N_{f}$-dependence of $\kappa_{\text {crit }}^{m}, \kappa_{\text {crit }}^{s}$ at $\tilde{y}_{N}=2.0$ as obtained on an $8^{4}$-lattice (open squares) and on a $6^{4}$-lattice (open circles). The analytical, finite volume, large $N_{f}$ predictions for the SYM-FM (SYM-AFM) phase transitions are represented by the dashed (dotted) lines. The dash-dotted lines are fits of the numerical data to linear functions as explained in the main text. In both plots $\tilde{\lambda}_{N}=0.1$ was chosen.

$L=4$ (dotted line), $L=8$ (dashed line), and $L=\infty$ (solid line). These analytically obtained lines perfectly describe the numerical results and one clearly observes the convergence of the numerical results to the analytically predicted $L=\infty$ line as the lattice size increases.

The $N_{f}$-dependence of the numerically obtained critical hopping parameters $\kappa_{\text {crit }}^{m}$ and $\kappa_{\text {crit }}^{s}$ is shown in Fig. $2 \mathrm{~b}$ for $\tilde{y}_{N}=2$. One clearly sees that for increasing $N_{f}$ the numerical results converge very well to the analytical finite volume predictions, as expected. It is interesting to note that the leading term in the finite $N_{f}$ corrections, i.e. the $1 / N_{f}$ contribution, seems to be the only relevant 
correction here, even at the small value $N_{f}=2$, as can be seen in Fig. $2 \mathrm{~b}$ by fitting the deviations to the function $f_{m, s}\left(N_{f}\right)=A_{m, s} / N_{f}$ with $A_{m, s}$ being the only free parameter. Furthermore, one observes that the critical hopping parameter $\kappa_{\text {crit }}^{m}$ is shifted towards larger values with decreasing $N_{f}$ while $\kappa_{\text {crit }}^{s}$ is shifted towards smaller values.

For an investigation of the model at large values of the Yukawa coupling constant see Refs. [9, 10].

\section{Outlook towards Higgs mass bounds}

In contrast to the previous discussion, where we considered the model mostly in the large $N_{f}$ limit, we now turn towards the physically interesting situation $N_{f}=1$. In order to investigate the model also at odd values of $N_{f}$ we have implemented a PHMC-algorithm, which we will discuss in detail in an upcoming publication.

The main goal here is to compute the cutoff $\Lambda$-dependence of the Higgs boson mass by fixing the top quark mass and the vacuum expectation value $v$ to their phenomenologically known values, i.e. $m_{\mathrm{top}}=175 \mathrm{GeV}$ and $v=246 \mathrm{GeV}$. From this dependence one can eventually determine an upper bound of the Higgs boson mass. The $v$ measured on the lattice has to be renormalized by the Goldstone renormalization factor $Z_{G}$ which can be obtained from the Goldstone-propagator $G\left(\hat{p}^{2}\right)$ according to

$$
G^{-1}\left(\hat{p}^{2}\right)=\frac{\hat{p}^{2}}{Z_{G}}
$$

with $\hat{p}^{2}$ denoting the squared lattice momentum. For the chosen setting $\left(\kappa_{N}=0.240, y_{N}=0.711, \lambda_{N}=\right.$ 1.0) we obtain $Z_{G}=0.9662 \pm 0.0001$ from the inverse Goldstone-propagator, plotted in Fig. 3a, and $\Lambda=(1684 \pm 14) \mathrm{GeV}$. In Fig. 3b we show one selected component of the fermion correlator $\left\langle\psi_{t_{1}} \bar{\psi}_{t_{2}}\right\rangle$ yielding the top mass $m_{\mathrm{top}}=(170 \pm 6) \mathrm{GeV}$ in accordance with the phenomenological value.

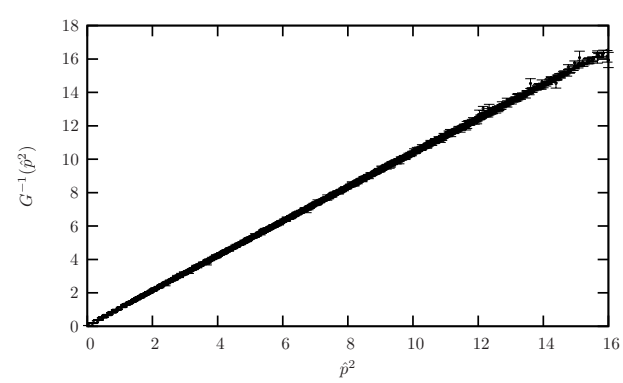

(a)

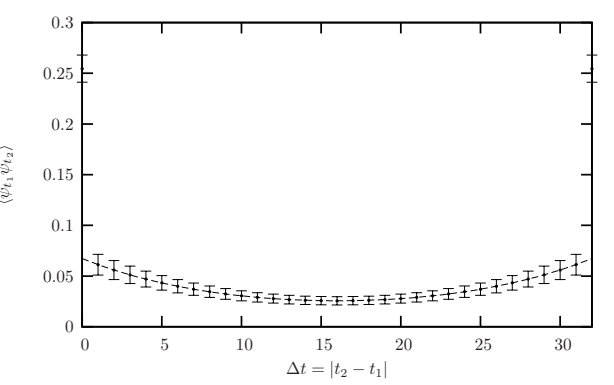

(b)

Figure 3: (a) Inverse Goldstone propagator $G^{-1}\left(\hat{p}^{2}\right)$ versus the squared lattice momentum $\hat{p}^{2}$ fitted to a linear function. (b) Fermion time slice correlator $\left\langle\psi_{t_{1}} \bar{\psi}_{t_{2}}\right\rangle$ versus distance in time direction $\Delta t=\left|t_{2}-t_{1}\right|$ fitted to a $\cosh$-function.

In the presented setup we chose the relatively large value of the quartic coupling constant $\lambda_{N}=1$, aiming for an upper Higgs mass bound. In Fig. 4a we present the corresponding result for the Higgs correlator $\left\langle\Phi_{t_{1}} \Phi_{t_{2}}\right\rangle$ versus $\Delta t$. We determine the Higgs mass by calculating the effective 
mass $m_{\mathrm{H}}^{\text {eff }}$ at several values of $\Delta t$ and finding its plateau value as shown in Fig. 4b. From this setup we find $m_{\mathrm{H}}=(565 \pm 15) \mathrm{GeV}$.

However, we remark that here we give only a first and very preliminary result towards our goal mentioned above. In particular, the value $L \cdot m_{\text {top }}=1.62$ is too small to determine the top quark mass reliably. Furthermore, the statistics (2500 configurations for the Higgs analysis) is still to low to obtain sufficiently precise results for the physical quantities of interest.

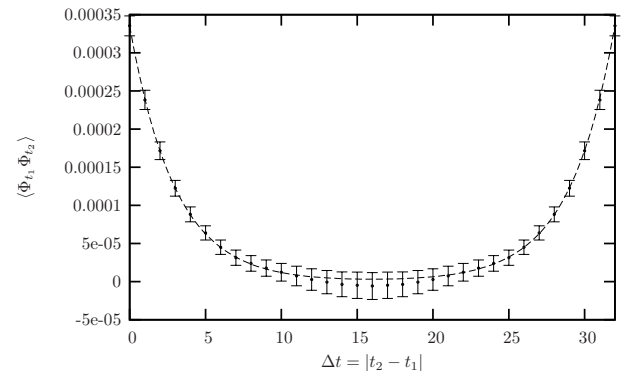

(a)

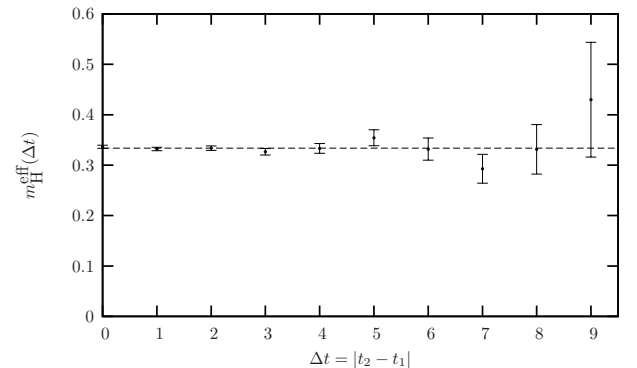

(b)

Figure 4: (a) Higgs time slice correlator $\left\langle\Phi_{t_{1}} \Phi_{t_{2}}\right\rangle$ versus $\Delta t$ fitted to a cosh-function. (b) Effective masses $m_{\mathrm{H}}^{\text {eff }}$ at $\Delta t$ fitted to plateau value $m_{\mathrm{H}}$.

\section{Acknowledgments}

We thank the "Deutsche Telekom Stiftung" for supporting this study by providing a Ph.D. scholarship for P.G. We further acknowledge the support of the DFG through the DFG-project Mu932/4-1. We are grateful to Joel Giedt, Julius Kuti, Michael Müller-Preussker, Erich Poppitz, and Christopher Schroeder for enlightening discussions and comments. In particular we want to express our gratitude to Julius Kuti for inviting P.G. to his group at the University of California, San Diego.

\section{References}

[1] K. Holland and J. Kuti. Nucl. Phys. Proc. Suppl. 129, 765-767 (2004).

[2] K. Holland. Nucl. Phys. Proc. Suppl. 140, 155-161 (2005).

[3] J. Smit. Nucl. Phys. Proc. Suppl. 17, 3-16 (1990).

[4] J. Shigemitsu. Nucl. Phys. Proc. Suppl. 20, 515-527 (1991).

[5] M. F. L. Golterman. Nucl. Phys. Proc. Suppl. 20, 528-541 (1991).

[6] A. K. De and J. Jersák. HLRZ Jülich, HLRZ 91-83, preprint edition (1991).

[7] M. Lüscher. Phys. Lett. B428, 342-345 (1998).

[8] P. H. Ginsparg and K. G. Wilson. Phys. Rev. D25, 2649 (1982).

[9] P. Gerhold and K. Jansen. JHEP 09, 041 (2007).

[10] P. Gerhold and K. Jansen. arXiv: 0707.3849 [hep-lat] (2007).

[11] H. Neuberger. Phys. Lett. B427, 353-355 (1998). 